\title{
Development of Tumor-Targeted Indocyanine Green-Loaded Ferritin Nanoparticles for Intraoperative Detection of Cancers
}

\author{
Leopoldo Sitia, Marta Sevieri, Arianna Bonizzi, Raffaele Allevi, Carlo Morasso, Diego Foschi, \\ Fabio Corsi, ${ }^{*}$ and Serena Mazzucchelli*
}

Cite This: ACS Omega 2020, 5, 12035-12045

Read Online

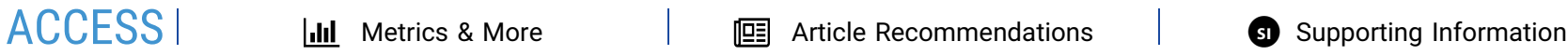

ABSTRACT: Indocyanine green (ICG) is a fluorescent dye with a strong emission in the near-infrared spectral range that allows deep signal penetration and minimal interference of tissue autofluorescence. It has been employed in clinics for different applications, among which the more interesting is certainly near-infrared fluorescence image-guided surgery. This technique has found wide application in surgical oncology for lymph node mapping or for laparoscopic surgery. Despite ICG being useful for tracking loco-regional lymph nodes, it does not provide any information about cancer involvement of such lymph nodes or lymphatic vessels, lacking any tumor-targeting specificity. However, the clinical need in surgical oncology is not only a specific tracking of metastatic nodes but also the intraoperative detection of micrometastatic deposits. Here, we have exploited a nanotechnological solution to improve ICG usefulness by its encapsulation in H-ferritin (HFn) nanocages. They are natural protein-based nanoparticles that exhibit some very interesting features as delivery systems in oncological applications because they display

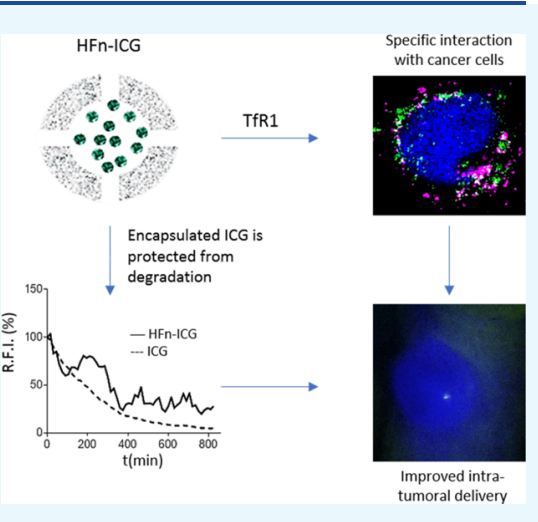
specific tumor homing. We show that HFn loaded with ICG exhibits specific uptake into different cancer cell lines and is able to deliver ICG to the tumor more efficiently than the free dye in an in vivo model of TNBC. Our results pave the way for the application of ICG-loaded HFn in fluorescence image-guided surgery of cancer.

\section{INTRODUCTION}

Indocyanine green (ICG) is an amphiphilic tricarbocyanine fluorescent dye with a strong fluorescence emission in the near-infrared (NIR) spectral range $(700-900 \mathrm{~nm})$. This allows deep penetration of the signal and minimizes interference of tissue autofluorescence. ${ }^{1}$ ICG has been used in clinics for more than 60 years, and it is approved for different applications, such as measuring cardiac output, ophthalmic angiography, and liver clearance evaluation. $^{2-4}$ More recently, ICG has also been employed in NIR fluorescence image-guided surgery (FGS). ${ }^{5}$ FGS has found application mainly in surgical oncology, where endoscopic administration of ICG has allowed successful lymph node mapping (LNM) both for colo-rectal cancer undergoing laparoscopic surgery and for breast cancer. ${ }^{6-8}$ More than 150 clinical trials are now being conducted using ICG for FGS in several types of cancer, including breast, gastric, colon, prostate, skin, and lung cancers, and promising results have already been reported. ${ }^{9}$

Despite feasibility and accuracy of ICG being demonstrated for real-time LNM and tracking of loco-regional lymph nodes, ICG does not provide any information about cancer involvement of such lymph nodes or lymphatic vessels, lacking any specific targeting toward tumor cells. ${ }^{10}$ Furthermore, clinical applications of ICG currently rely on knowledge about primary tumor localization because a subdermal or submucosal injection is required for LNM. ${ }^{11}$ However, surgeons would need not only a specific tracking of metastatic nodes but also to detect intraoperatively micrometastatic deposits (i.e., tumor deposits not associated to lymph nodes in rectal cancer or microscopic peritoneal carcinomatosis in gastric or ovaric cancer). ${ }^{12}$

Therefore, in the attempt of developing an in vivo fluorescent tracer able to specifically label tumor tissue, the exploitation of a tumor-targeted ICG-based nano-delivery system could represent an interesting tool. Several nanotechnological approaches have been proposed for ICG delivery, mainly using micelles, ${ }^{13}$ polymeric nanoparticles, ${ }^{14-17}$ silica nanoparticles, and liposomes, ${ }^{18-20}$ but their tumor specific accumulation has been hardly demonstrated. Indeed, in these ICG-based nanoparticles, dye accumulation into the target site is mainly triggered by the enhanced permeation effect (EPR). Therefore, ICG accumulates also into tissues surrounding the tumor mass, thus preventing the correct discrimination between non-cancerous and cancerous tissues. ${ }^{21}$ Despite this, these strategies have allowed to improve ICG

Received: January 17, 2020

Accepted: April 29, 2020

Published: May 20, 2020 
fluorescence stability and increasing circulation time, solving some issues that actually prevent free ICG intravenous administration, such as the quick loss of fluorescence in aqueous media and the strong quenching effect observed at higher dye concentrations. ${ }^{21}$ Here, we try to combine the advantages related to ICG nanoformulation with tumor target specificity thanks to the exploitation of $\mathrm{H}$-ferritin ( $\mathrm{HFn}$ ) nanocages loaded with the dye for intraoperative detection of tumor. $^{21-23}$

HFn is a protein nanocage that displays a cave sphere structure of $12 \mathrm{~nm}$ in diameter constituted by 24 selfassembling subunits. ${ }^{24,25}$ From the nanotechnological point of view, HFn exhibits some very interesting features combining a low toxicity because of its protein nature with a particularly high tumor homing ability. ${ }^{26,27}$ Indeed, HFn binding and internalization in cells is mediated by the transferrin receptor-1 (TfR1), which is highly overexpressed in cancers. ${ }^{28}$ Moreover, HFn is highly stable in biological fluids and extremely resistant to high temperatures (up to $80^{\circ} \mathrm{C}$ ) and to acidic conditions: at low $\mathrm{pH}, \mathrm{HFn}$ subunits are disassembled, with the possibility of loading different cargoes inside the cavity, while they are naturally reassembled when $\mathrm{pH}$ is brought back to neutral. ${ }^{29}$ These characteristics make HFn a very promising nanocarrier in oncological applications. Several HFn-based nanodrugs have been developed for cancer treatment at a preclinical level with excellent results in terms of specific tumor recognition, improved drug penetration, optimized subcellular targeting, increased activity with lower side effects, and reduced onset of chemoresistance. $^{30-33}$

In this work, we prepared ICG loaded HFn nanocages (HFn-ICG) and we assessed their uptake into different gastric, breast, and colo-rectal cancer cell (CC) lines. Moreover, we evaluated HFn-ICG biodistribution in a preclinical model of breast cancer demonstrating that ICG can be specifically internalized into tumor cells and delivered to the tumor mass thanks to the strong targeting affinity of HFn with TfR1. Our results prove the suitability of HFn-ICG as an in vivo nanotracer for FGS and intraoperative detection of tumors.

\section{RESULTS AND DISCUSSION}

HFn Suitability as an ICG Delivery Vector. In the attempt of supporting surgeons in intraoperative tumor and metastasis localization, the exploitation of a nanoparticle-based ICG delivery system able to specifically label cancer tissues would have a relevant translational impact. Among the plethora of possibilities offered by nanotechnology, we have reasoned that HFn nanocages could represent the most valuable solution. Indeed, these nanoparticles displayed (1) a cave sphere structure loadable with dyes, (2) good biocompatibility and solubility profile due to its protein nature, and (3) a natural tumor homing.

In addition to this, we have also hypothesized that tumortargeted ICG nanoparticles could be applied in FGS of certain kinds of tumors, such as breast, colo-rectal, and gastric cancers. Therefore, we have established a panel of human CC lines, selecting MCF-7, HCC1937, MDA-MB 231, and MDA-MB 468 among breast CC lines, while SNU-16, HT-29, and HCT116 as models of gastric and colo-rectal cancer, respectively. Because HFn interaction and internalization in CCs is mediated by its capability to specifically recognize the TfR1 overexpressed in a lot of cancers, ${ }^{28}$ we have first evaluated TfR1 expression in the surface of these cell lines by flow cytometry.

Surface expression of TfR 1 reported in Figure $1 \mathrm{a}, \mathrm{b}$ evidenced that CC lines included in our panel could be
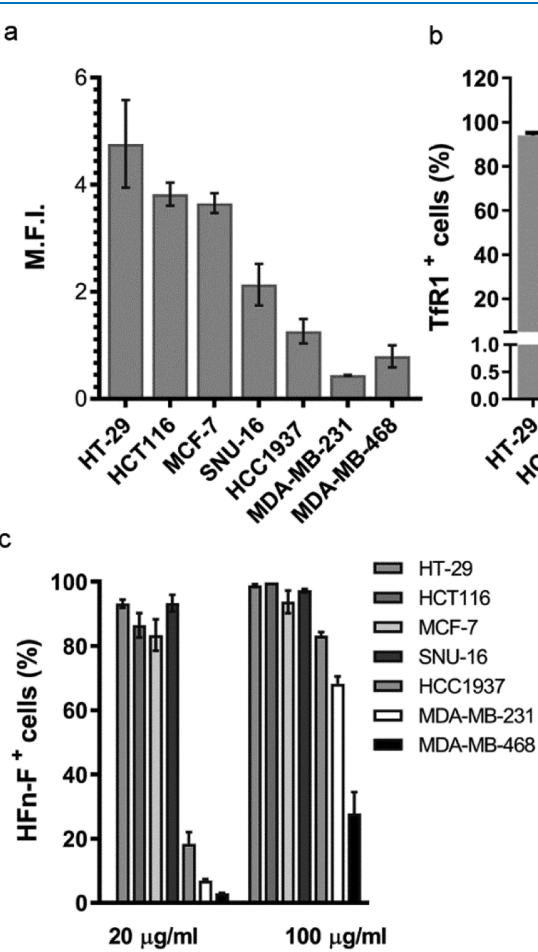

b

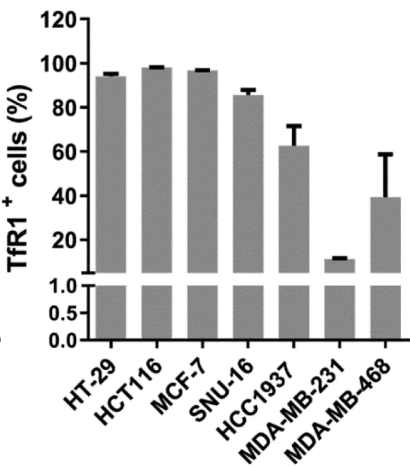

d
Figure 1. HFn specific interaction with CC lines. TfR1 expression of $\mathrm{CC}$ lines tested by cytofluorimetry expressed as mean fluorescence intensity (a) and as percentage of positive cells (b). Cells immunodecorated with the anti-mouse secondary antibody conjugated with Alexa Fluor 488 were used to set the gate on viable cells, on singlets, and the region of positivity. (c) HFn-F binding with CCs. Cells were incubated $2 \mathrm{~h}$ at $4{ }^{\circ} \mathrm{C}$ in PBS buffer and $0.3 \%$ BSA with different amounts of HFn-F (20 and $100 \mu \mathrm{g} / \mathrm{mL})$. Cells were processed for flow cytometry using untreated cells to set the positive region and the singlet gate. (d) Competition assay in HT-29 cells (high TfR1 expression) incubated with $500 \mu \mathrm{L}$ of HFn-F $(20 \mu \mathrm{g} /$ $\mathrm{mL})$ at $4{ }^{\circ} \mathrm{C}$ for $2 \mathrm{~h}$ with or without an excess of unlabeled HFn (1 $\mathrm{mg} / \mathrm{mL}$ ) as competitor. Cells were then detached and treated for flow cytometry. Untreated cells have been used to set the singlet gate and the positive region. Data are reported as average \pm S.D. of three independent experiments and expressed as panel (a), mean fluorescence intensity (M.F.I., $\left.\times 10^{5}\right)$; panel (b), percentage of cells in the positive region to $\mathrm{HFn}-\mathrm{F}$ fluorescence and; panel (c), relative fluorescence intensity (R.F.I., \%).

distinguished into two groups: one that displays high TfR1 expression and one characterized by a lower TfR 1 expression: HT-29, HCT116, MCF-7, and SNU-16 exhibited 3-5 fold higher TfR1 expression than HCC1937, MDA-MB 231, and MDA-MB 468. To better define TfR1 overexpression in the $\mathrm{CC}$ lines used in the present study, we compared our results with data we previously published, in which the low TfR1 expressing CCs used here displayed a TfR1 expression higher than the one observed in a healthy control cell line, such as human umbilical vein endothelial cells, which among healthy cells is characterized by a relatively high TfR1 expression. ${ }^{31}$ 
a

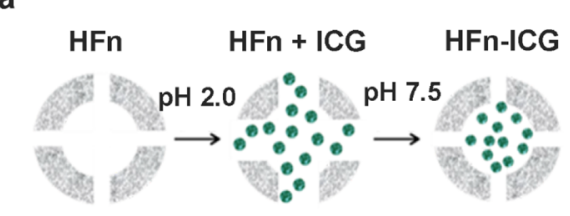

b

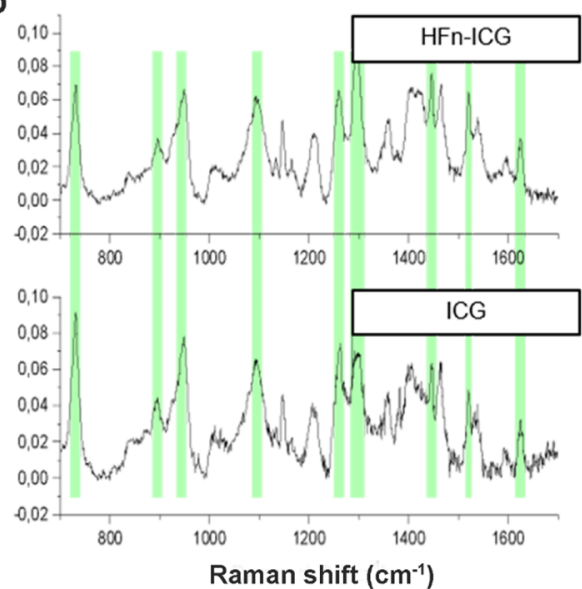

C
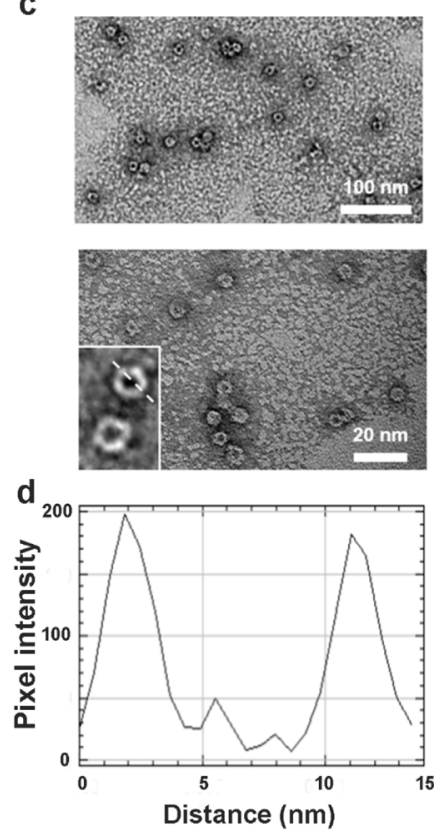

Figure 2. Development and characterization of ICG-loaded HFn nanocages. (a) Schematic representation of the pH-dependent disassembly/ reassembly method used for HFn loading with ICG. ICG is represented in green and HFn is represented in gray. (b) Raman characterization of HFn-ICG nanoformulation; the peaks of free ICG (green highlights) are also found in HFn-ICG samples, meaning that the dye was efficiently loaded within the nanoformulation. (c) TEM images of HFn-ICG at different magnifications and (d) a representative profile plot confirming the size of the nanoassembly, with an inner and outer shells of approximately 8 and $12 \mathrm{~nm}$, respectively.

Moreover, we evaluated TfR1 expression in another cell line of normal mammary tissue, (MCF-10A) where we obtained $0.2 \%$ of $\mathrm{TfR}^{+}$cells (data not shown). This is much lower than all other CCs tested here that were all between 12\% (MDAMB 231) and 98\% (HCT116) (Figure 1b).

Then, we have evaluated the binding of fluorescently labeled HFn nanocages with our panel of CCs (Figure 1c). FITC was covalently conjugated to HFn (HFn-F) by amide-bond formation between Lys residues on HFn and the carboxyl group on FITC to avoid issues related to dye leakage during incubation with cells and to analyze only cells that were actually bound to HFn excluding those simply stained by the free dye. CCs were incubated for $2 \mathrm{~h}$ at $4{ }^{\circ} \mathrm{C}$ with two different amounts of HFn $-\mathrm{F}(20$ and $100 \mu \mathrm{g} / \mathrm{mL})$ and then analyzed by flow cytometry. Interestingly, we found that the panel of CCs could be easily divided into the same two groups observed when studying their TfR1 expression. CCs with high expression of TfR1 (HT-29, HCT116, MCF7, and SNU-16) displayed high percentages of binding already at the lowest HFn-F concentration (i.e., $20 \mu \mathrm{g} / \mathrm{mL}$ ). Here, $80-90 \%$ of cells were positive to HFn-F staining at the low dose, while these percentages reached $100 \%$ at the higher dose of $100 \mu \mathrm{g} / \mathrm{mL}$. On the contrary, HCC1937, MDA-MB 231, and MDA-MB 468, which exhibited lower TfR1 expression, showed much lower interaction with HFn-F. Once again, we could observe a dose-dependent recognition, as demonstrated by the increase in binding percentages when incubating cells with $100 \mu \mathrm{g} / \mathrm{mL}$ of HFn-F. Binding assay performed with a cell line of normal mammary tissues (i.e., MCF10A) reported in Figure S1 suggests the suitability of HFn as the tumor-targeted delivery system.

To further characterize HFn-F cell interaction and to verify if this is actually mediated by TfR 1 expression, we ran a competition binding assay using HT-29 cell line. Keeping cells at $4{ }^{\circ} \mathrm{C}$ to avoid endocytosis, we first incubated cells with a 50 fold excess of unlabeled HFn $[1 \mathrm{mg} / \mathrm{mL}$ in phosphate buffered solution (PBS)], as a competitor. Then, we added HFn-F at a concentration of $20 \mu \mathrm{g} / \mathrm{mL}$ and evaluated the binding efficiency. As it is shown in Figure 1d, we observed a strong competition with a $60 \%$ binding reduction, which confirms the specificity of the interaction between CC and HFn nanocages. One more time, the crucial role of TfR1 in mediating CCsHFn interaction has been stated, demonstrating HFn suitability as an ICG delivery vector.

HFn Efficiently Encapsulates ICG and Stabilizes Its Fluorescence Properties. To perform HFn loading with ICG, we have followed the procedure illustrated in Figure 2a and widely used in literature to load drugs and other dyes into ferritin nanocages. ${ }^{34,35}$ Briefly, HFn was unfolded lowering the $\mathrm{pH}$ to 2.0 , and then, the protein was refolded bringing back the $\mathrm{pH}$ to neutrality. ICG was added at the beginning of the HFn refolding process and incubated at room temperature (RT) for about $2 \mathrm{~h}$. Non-encapsulated ICG has been removed by size exclusion chromatography, loading the formulation in Zeba spin desalting columns.

Encapsulation of ICG was confirmed by Raman spectroscopy. In Figure $2 b$, the spectra of ICG in solution after encapsulation in HFn nanocages are reported. As it can be seen in the figure, typical peaks of free ICG (lower panel) were also detected in the spectrum of HFn-ICG (higher panel), thus confirming the effective loading of the dye into HFn nanocages.

To verify the integrity of HFn after ICG loading, we have analyzed their structure by transmission electron microscopy (TEM) (Figure 2c). The images clearly evidenced both the inner cavity of the nanocages and the circular outer shape and confirmed HFn-ICG structural integrity, with the single subunits of the protein that can be observed in the zoomed 

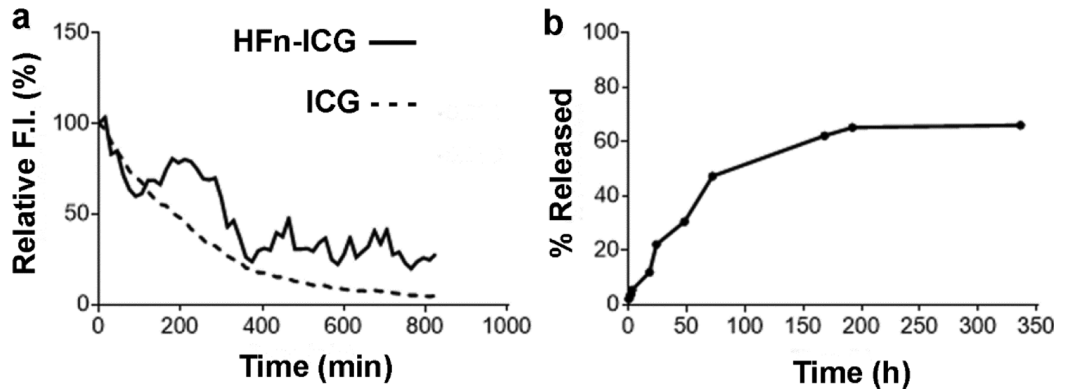

Figure 3. Stabilization of ICG upon nanoformulation. (a) Evaluation of the fluorescence lifetime of HFn-ICG (continuous line) and free ICG (dashed line) in $\mathrm{ddH}_{2} \mathrm{O}$ by spectrofluorimetry. (b) ICG release from HFn-ICG evaluated by spectrofluorimetry after 14 days dialysis in dd $\mathrm{H}_{2} \mathrm{O}$ at $4{ }^{\circ} \mathrm{C}$.
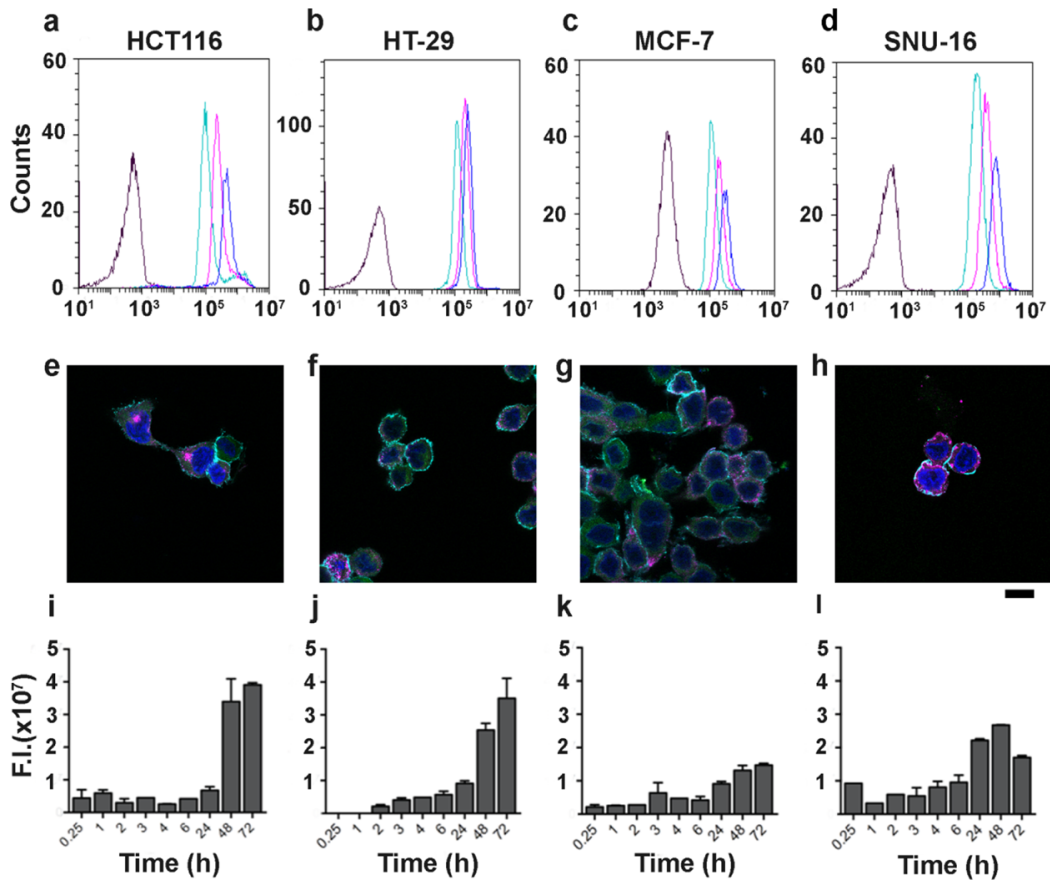

Figure 4. HFn-ICG interaction with TfR $1^{\text {high }}$ CCs. $(\mathrm{a}-\mathrm{d})$ Representative flow cytometry plots showing cell binding with 20,50 , and $100 \mu \mathrm{g} / \mathrm{mL}$ of HFn-ICG (light green, purple, and blue curves, respectively, black curves $=$ control cells) after $2 \mathrm{~h}$ incubation at $4{ }^{\circ} \mathrm{C}$. $(\mathrm{e}-\mathrm{h}) \mathrm{Representative}$ confocal microscopy images of cells incubated with HFn-ICG for $2 \mathrm{~h}$ at $4{ }^{\circ} \mathrm{C}$ to evaluate binding and TfRl colocalization (blue = cell nuclei stained with DAPI, cyan = cell membrane, green $=$ HFn-ICG, and purple $=\alpha$ TfR1 antibody staining). (i-1) Cellular uptake of HFn - ICG NPs evaluated by IVIS analysis, after incubation at $37^{\circ} \mathrm{C}$ for different time points. High TfR1 expression lead to high binding, diffused colocalization with TfR1 (white spots) and strong HFn-ICG uptake.

images of the lower-left corner of the panel. We then analyzed the profile plot of the nanocages to confirm the final size of the structure (Figure 2d). As expected, we obtained nanocages with an inner size of $8 \mathrm{~nm}$ and an outer diameter of $12 \mathrm{~nm}$, thus confirming the size of HFn reported in literature. ${ }^{36}$

A quantitative analysis of ICG loading efficiency was performed by spectrofluorimetry. First, we have measured the fluorescence of the nanocomposites and compared it with a standard curve of the dye. To obtain a reliable standard curve, we have evaluated ICG fluorescence in different media, as it is known that its emission properties radically change depending on the medium where the dye is dissolved. ${ }^{37}$ As it can be seen in Figure S2, the fluorescence yield in water and PBS is much lower than in media containing lipids and proteins, such as Dulbecco's modified Eagle medium (DMEM), plasma, and milk. Moreover, a linear correlation between ICG fluorescence and dye concentration could be obtained only working in complex media (i.e., DMEM, plasma, and milk). Milk was particularly reliable in terms of measurement reproducibility and sensitivity, so we have decided to use it to perform the quantification of ICG encapsulation. We have prepared a standard curve by diluting different concentrations of ICG in milk (Figure S3, panel a), and we have observed a maximum value of fluorescence emission at around $10-20 \mu \mathrm{M}$ ICG solution. Above this concentration, the peak shifted from 805810 to $820 \mathrm{~nm}$, and the fluorescence intensity started to decrease because of a strong quenching effect, as also reported in literature. ${ }^{23}$ To quantify the concentration of the dye inside the HFn-ICG preparation, we have extracted ICG from the nanocages with an acetonitrile- $\mathrm{ddH}_{2} \mathrm{O}$ solution (I), diluted in milk (II), and we have compared the fluorescence values with the standard curve (III). We have tested different dilutions to avoid quenching effect. The average concentration of loaded dye was $1.01 \pm 0.07 \mathrm{mg} / \mathrm{mL}$, corresponding to a loading efficiency of $40.4 \pm 4.5 \%$ of the initial dye that was reacted with HFn. The effectiveness of ICG encapsulation is strongly 

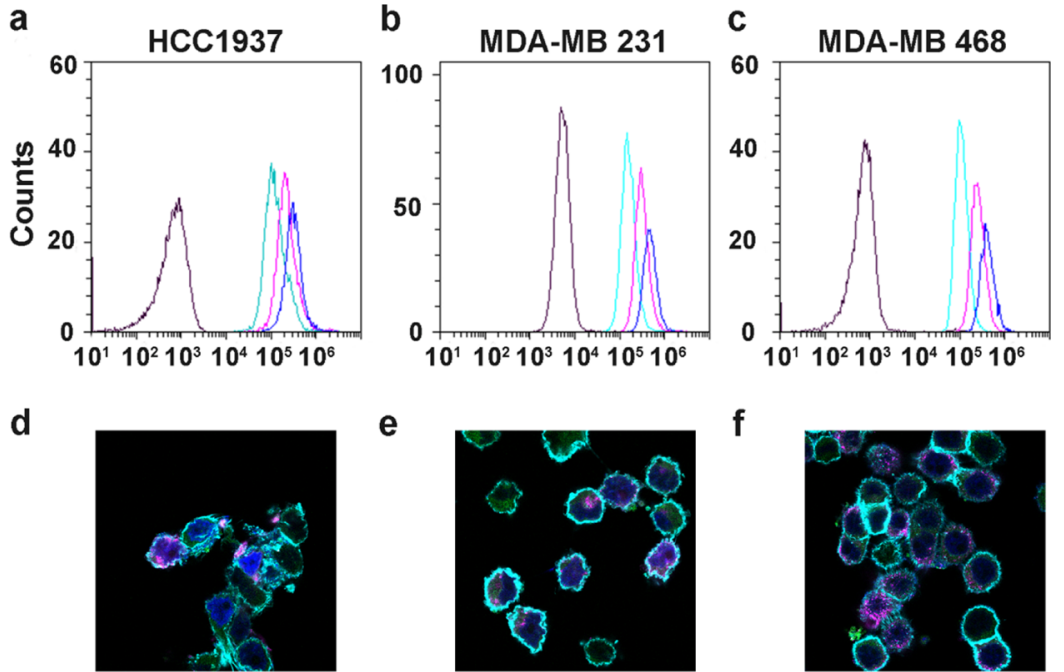

e
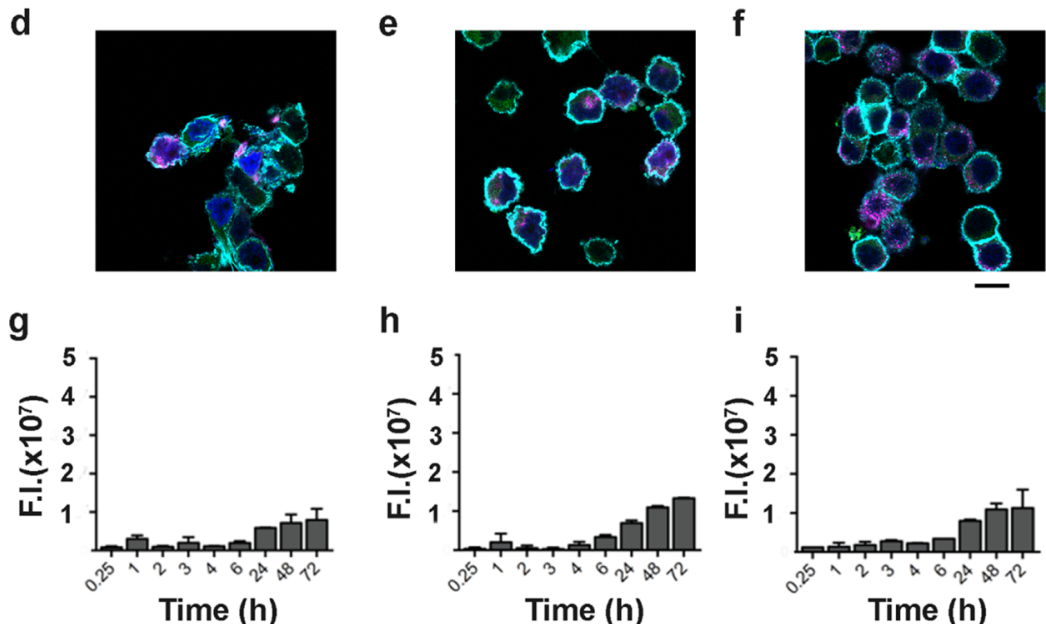

Figure 5. HFn-ICG interaction with TfR $1^{\text {low }}$ CCs. (a-c) Representative flow cytometry plots showing cell binding with 20 , 50 , and $100 \mu$ g/mL of HFn-ICG (light green, purple, and blue curves, respectively, black curves = control cells) after $2 \mathrm{~h}$ incubation at $4{ }^{\circ} \mathrm{C}$. ( $\mathrm{d}-\mathrm{f}$ ) Representative confocal microscopy images of cells incubated with HFn-ICG for $2 \mathrm{~h}$ at $4{ }^{\circ} \mathrm{C}$ to evaluate binding and TfR 1 colocalization (blue $=$ cell nuclei stained with DAPI, cyan = cell membranes, green = HFn-ICG, and purple = $\alpha$ TfR1 antibody staining). (g-i) Cellular uptake of HFn-ICG evaluated by IVIS analysis, after incubation at $37^{\circ} \mathrm{C}$ for different time points. Low TfR1 expressions lead to low binding, low uptake, and low colocalization with TfR1.

supported also by some indirect data. As shown in Figure S3 (panel b), after acetonitrile extraction of the dye, the fluorescence intensity of ICG increased and the maximum peak shifted from 820 to $810 \mathrm{~nm}$. This suggests that the dye was entrapped inside HFn and its fluorescence quenched. Moreover, the average size observed for HFn-ICG nanocages did not vary as compared to empty HFn, proving that the dye is encapsulated inside HFn cavity and not simply adsorbed on the protein shell. These data coupled with Raman spectra demonstrating the presence of ICG in the final product strongly prove that the dye has been efficiently encapsulated inside the nanocages.

After verifying that the dye had been encapsulated in the nanocages, we have tested whether the protein shell would stabilize its fluorescence properties. In fact, ICG low fluorescence stability is a big issue with the use of ICG in clinical practice. ${ }^{22}$ To verify this, we have measured the fluorescence decay of both HFn-ICG and ICG until we reached the limit of detectability for the free dye (about 800 min) (Figure 3, panel a). At this time point, the fluorescence of the encapsulated ICG was still $40 \%$ of the initial value with a much slower decay rate $(-0.392$ fluorescence units/minute for HFn-ICG as compared to -0.590 fluorescence units/minute for free ICG). The two curves are significantly different because the free ICG displayed a regular decay profile, while the nanoformulated one is more irregular, suggesting that only the fraction of dye released over time is degraded, while the dye that is still encapsulated inside the protein cavity is protected from degradation, thus improving ICG fluorescence lifetime. These data confirm the role of HFn encapsulation in stabilizing ICG fluorescence.

To further characterize HFn-ICG, we have analyzed the kinetics of ICG release from HFn nanocages by dialysis. As it can be seen in Figure $3 b$, the profile is very regular, with no evident burst effect during the first hours of incubation, and around 50\% of the dye is released from HFn during the first 72 $\mathrm{h}$, suggesting a good stability of the complex. After this time point, the release slows down, reaching a plateau after 1 week of incubation, when $66 \%$ of the dye has been released. We continued the analysis for one more week, but no further increase in fluorescence was recorded, meaning that no more ICG has been released in solution. Representative pictures of the samples collected throughout the dialysis are shown in Figure S4. Interestingly, HFn-ICG collected from inside the dialysis membrane $\left(t 14_{\text {in }}\right)$ are still of a bright green, almost as intense as the one at the beginning of analysis $\left(t 0_{\text {in }}\right)$, thus confirming the high stability of the complex.

Dose-Dependent HFn-ICG Binding with CCs. To further characterize the interaction of HFn-ICG with our panel of CCs, we performed a binding assay with increasing concentrations of loaded nanocages (Figures $4 a-d$ and $5 a-c$ ). Similar experiments with free ICG have not been performed because ICG undergoes non-specific binding and internalization. We incubated cells with three different HFn-ICG concentrations $(20,50$, and $100 \mu \mathrm{g} / \mathrm{mL})$, and we studied by flow cytometry the fluorescence distribution associated with cell interactions. Results demonstrated that the binding with cells is dose-dependent in all cell lines, as the mean 

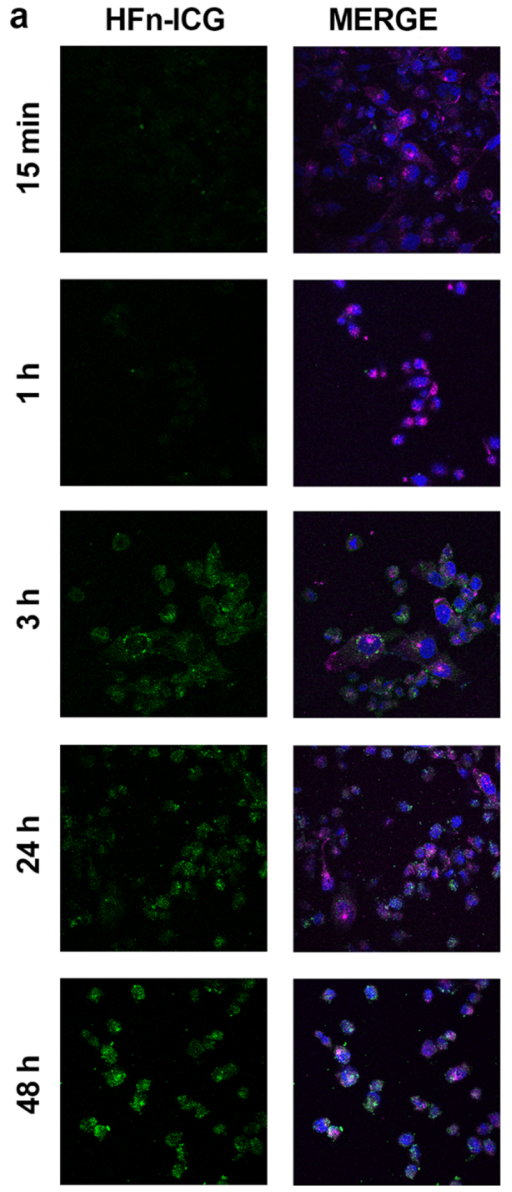
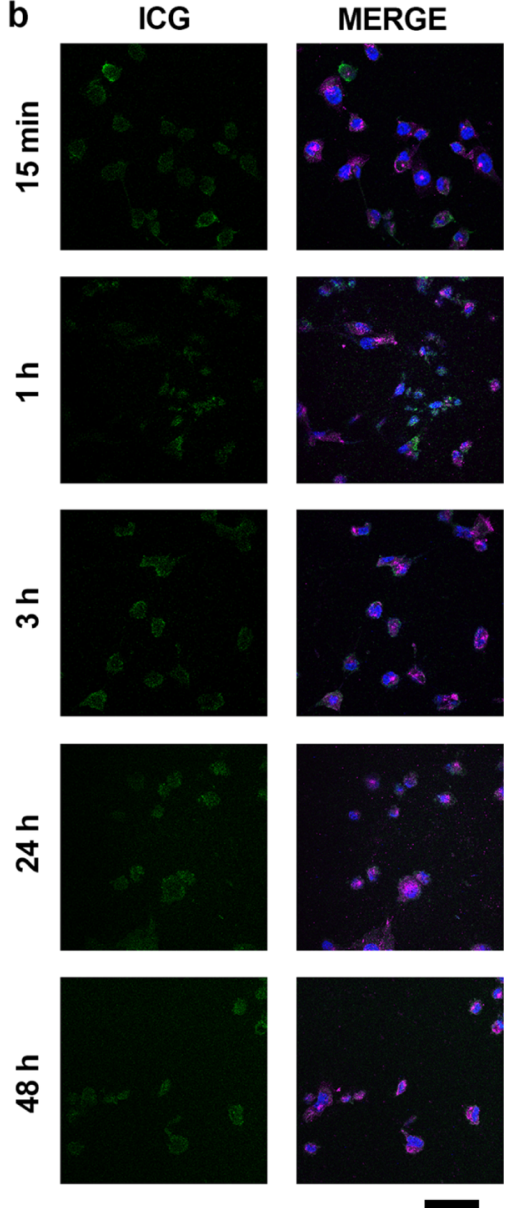

Figure 6. Colocalization of HFn-ICG and TfR1. (a,b) Confocal images of MDA-MB 231 cells incubated 15 min, $1,3,24$, and $48 \mathrm{~h}$ at $37{ }^{\circ} \mathrm{C}$ in complete cell culture medium with HFn-ICG or free ICG [green; $50 \mu \mathrm{g} / \mathrm{mL}$; panel $(\mathrm{a}, \mathrm{b})$, respectively]. Nuclei were stained with DAPI (blue). TfR1 was recognized with anti-TfR1 antibody (Abcam) and labeled with an anti-rabbit secondary antibody conjugated with Alexa Fluor 488. Scale bar $=50 \mu \mathrm{m}$.

fluorescence intensity increases up to the concentration of 100 $\mu \mathrm{g} / \mathrm{mL}$. As expected, in CCs with high TfR1 expression, we reached $100 \%$ of stained cells already at the lowest concentration of ICG (i.e., HFn-ICG at $10 \mu \mathrm{g} / \mathrm{mL}$ ) (Figure $4 a-d)$, despite the mean fluorescence intensity still increasing in a dose-dependent manner, suggesting that the binding is not saturated yet. Even more interestingly, we observed a dosedependent increase in binding, also in cells with low TfR1 expression (Figure $5 \mathrm{a}-\mathrm{c}$ ). This, together with the low but specific binding observed with HFn-F (Figure 1b) suggests that even a low TfR1 expression is enough to obtain a specific ICG label of CCs, evidencing the suitability of HFn as a nanodelivery system for ICG.

TfR1 is Involved in HFn-ICG Uptake in CCs. Involvement of TfR1 in mediating binding and uptake of HFn-ICG nanocages has been assessed by confocal microscopy. CCs were incubated with HFn-ICG for $2 \mathrm{~h}$ at $4{ }^{\circ} \mathrm{C}$ and stained with an anti-TfR 1 antibody. Representative images of CCs with high levels of TfR1 are reported in Figure $4 \mathrm{e}-\mathrm{h}$, where the strong purple signal, observed in almost all analyzed cells, confirmed the high expression of the receptor. Here, the HFn-ICG signal (green) is mostly associated with cell membranes and partially diffused in peripheral areas of the cytoplasm. Moreover, the majority of the HFn-ICG signal seems to colocalize with TfR1 staining with a dotteddistribution that reminds association with TfR1 rich endo- somes that are involved in iron metabolism. ${ }^{38}$ The colocalization around cell membranes was somehow expected. Even if incubation was performed at $4{ }^{\circ} \mathrm{C}$, a portion of nanocages seem to be distributed intracellularly. This might be due to the strong interaction with TfR 1 and the fast uptake kinetics that are characteristics of this receptor. ${ }^{39}$

In Figure 5d-f, HFn-ICG interaction with low TfR1 expressing CCs (HCC1937, MDA-MB 231, and MDA-MB 468) has been depicted. In these CCs, not all cells were TfR $1^{+}$, confirming that the low expression obtained by flow cytometry (Figure 1b). Quite interestingly, HFn-ICG signal was almost exclusively found around $\mathrm{TfR} 1^{+}$cells, where strong accumulation on cell membranes and very rare colocalization spots were observed inside the cells. On the contrary, almost no HFn-ICG interaction was observed with TfR $1^{-}$cells.

Further indirect confirmation of the TfR 1 involvement in CCs uptake of HFn-ICG was obtained by studying the intracellular accumulation of ICG by IVIS Lumina II, for different time points (Figures $4 \mathrm{i}-\mathrm{l}$ and $5 \mathrm{~g}-\mathrm{i}$ ). To avoid fluorescence quenching problems normally observed at high ICG concentrations, we extracted the dye with a 1:1 solution of acetonitrile and water. This allowed us to release the dye from intracellular vesicles, where it could reach a relatively high local concentration into the acetonitrile-based solution and maximize its fluorescence emission. In all CCs, we obtained a time-dependent intracellular uptake of HFn-ICG, where the 
a

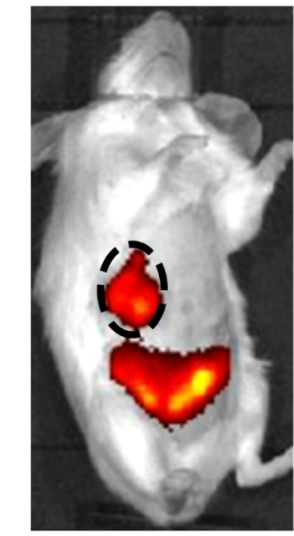

c

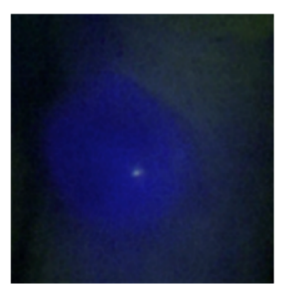

b
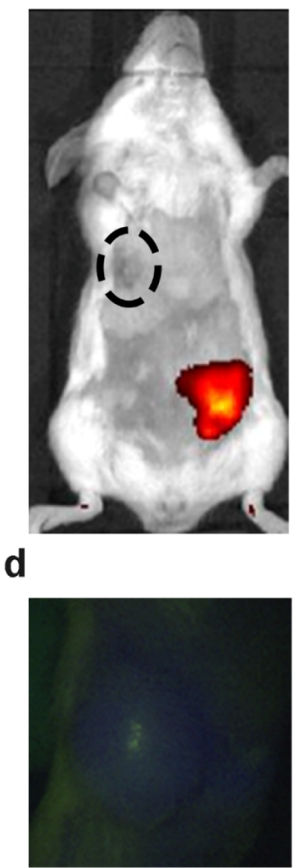

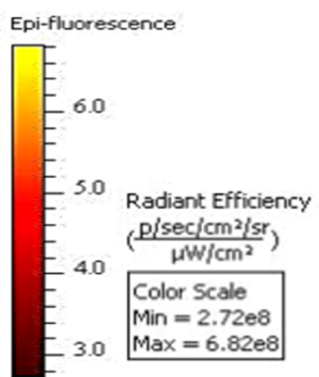

e

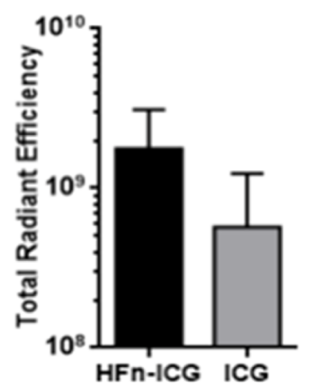

Figure 7. In vivo tumor targeting of HFn-ICG and ICG. In vivo targeting of HFn-ICG (a,c) and ICG (b,d) was evaluated in tumor-bearing mice, $6 \mathrm{~h}$ after I.V. administration by IVIS Lumina II and by KARL STORZ NIR/ICG endoscopic system. (e) Imaging analysis of data obtained by IVIS Lumina II allowed us to quantify the dye in the tumor area (black dotted ROI). Fluorescence was higher in HFn-ICG than in free ICG-treated mice, but the difference was not statistically significant $(p$-value $=0.1112)$. Color scale expressed as total radiant efficiency $\left(\times 10^{8}\right), n=3$.

fluorescent signal was detectable already after $15 \mathrm{~min}$ of incubation. The signal slightly increased over time, with minimal differences up to $6 \mathrm{~h}$, while it reached the highest intensity after 48 and $72 \mathrm{~h}$ of incubation. As expected, we have noticed that TfR1 high expression led to a 2-5 times higher particle uptake than in low TfR1 expressing cells. The maximum signal has been reported in HCT116 and HT-29, while the lowest fluorescence has been revealed in HCC1937 cells.

Nanoformulation Affects ICG Uptake and Accumulation. We further studied intracellular uptake of ICG by incubating MDA-MB 231 cells with both free and nanoformulated ICG and observing the distribution of the fluorescence signal over time by confocal microscopy. Among all the tested CCs, we selected MDA-MB 231 to prove that HFn encapsulation could significantly improve intracellular uptake of ICG even in cells, where TfR 1 is not at its maximum expression. The fluorescence signal of HFn-ICG (green) gradually increased (Figure 6a): it remained very low within the first hour of incubation, while it started to be clearly visible after the third hour of incubation. The dotted distribution of intracellular HFn-ICG seems to confirm a vesicle-mediated uptake mechanism, with subsequent perinuclear accumulation, as expected for a transferrin mediated uptake, which is characterized by binding with TfR 1 on the cell membrane, clathrin-mediated endocytosis, accumulation into late endosomes, and then recycling to the cell membranes. ${ }^{40}$ On the contrary, the uptake pattern observed for free ICG was completely different (Figure 6b). The signal was already visible inside the cells after $15 \mathrm{~min}$ of incubation and, opposite to HFn-ICG, it did not seem to increase over time. This might be due to the low uptake of the free dye in the cells and to a fast degradation of the molecule with consequent fluorescence loss. As expected, no perinuclear ICG accumulation and no colocalization with TfR1 were observed for ICG, suggesting that the dye does not follow the same pathway of HFn-ICG.

To corroborate these observations, we have decided to perform also a pilot experiment in vivo with the aim to demonstrate the improved uptake performances of nanoformulated ICG in comparison to ICG free. To reach this goal, we have used an in vivo ortotopic and syngeneic model of murine breast cancer, obtained by injection of $4 \mathrm{~T} 1$ cells into the mammary fat pad of Balb/C female mice. This model has been selected because $4 \mathrm{~T} 1$ cells display low TfR 1 expression ${ }^{41}$ and low binding with HFn nanocages as previously demonstrated. ${ }^{30}$ Therefore, obtaining good targeting results with this model could reasonably allow us to hypotesize that better results could be obtained with tumors characterized by higher expression of TfR 1 and better HFn recognition. Indeed, a different but non-significant trend in tumor accumulation of ICG has been detected after administration in mice treated with the free dye or with HFn-ICG, as reported in Figure 7 ( $p$-value $=0.1112)$. By in vivo fluorescence imaging performed $6 \mathrm{~h}$ after administration, we observed a strong HFn-ICG signal localized in the tumor mass, while free ICG did not display specific intratumor accumulation. The free dye was only detected in the liver and the intestine tract. This was expected as it is widely known that free ICG is rapidly metabolized in the liver and released in the intestine together with bile salts to be excreted in the stool. ${ }^{42}$ The fluorescent signal observed in the lower abdominal area in HFn-ICGtreated mice is most likely due to both the non-tumor accumulated HFn nanocarrier that is being metabolized and to free dye that has been partially released from HFn in the meantime. 
Twenty four hours after administration, no fluorescence was observed either in HFn-ICG- or free-ICG treated mice, with no undesirable accumulation of either the carrier and the dye (Figure S6).

Overall, the improved fluorescence properties, the high cellular uptake efficacy, and the ability of targeting the tumor mass in vivo suggest the great potential of HFn-ICG as an exploitable delivery agent for ICG in solid tumors.

\section{CONCLUSIONS}

ICG is routinely used in surgical oncology for LNM mapping and FGS with good results. However, ICG labels cancer in a not specific way, limiting the FGS application in tumor with well-known localization. Here, tumor specific delivery of ICG was successfully achieved in a wide panel of CC lines using HFn as the tumor targeted delivery system for ICG, allowing its use to detect, label, and potentially remove also unknown micrometastatic tumor deposits. Indeed, HFn is naturally internalized by CCs thanks to TfR1-mediated endocytosis and displays an inner cavity easily loadable with hydrophilic drugs or contrast agents. Therefore, ICG can be loaded into HFn with a loading efficiency of more than $40 \%$, and it can be delivered into a wide panel of CCs depending on their TfR1 expression, allowing achievement of tumor targeting capability to ICG. Moreover, nanoformulation in HFn nanocages improves the fluorescence properties of ICG and avoids its quick degradation, increasing retention time in the body.

These promising results confirm the ability of HFn to deliver ICG specifically to the tumors and pave the way for the application of HFn-ICG in FGS of cancer.

\section{MATERIALS AND METHODS}

Development of ICG-Loaded-HFn Nanoparticles. HFn was purchased from MoLiRom s.r.l. (Rome, Italy). For in vitro preliminary studies with the panel of CCs, HFn was labeled with fluorescein isothiocyanate Isomer I (Sigma-Aldrich S.r.l., CAS Number: 3326-32-7, Milan, Italy) according to the manufacturer's protocol. ICG has been nanoformulated exploiting the ability of HFn to disassemble and reassemble its quaternary structure in response to changes in $\mathrm{pH}$. First, a mixture of HFn $(0.5 \mathrm{mg} / \mathrm{mL})$ dissolved in $0.15 \mathrm{M} \mathrm{NaCl}$ was brought to $\mathrm{pH} 2.0$ for $15 \mathrm{~min}$ at $\mathrm{RT}$ to disassemble the protein cage. Then, the $\mathrm{pH}$ was brought back to neutrality. In the meantime, ICG powder Verdye (25 mg; Diagnostic Green $\mathrm{GmbH}$, Aschheim-Dornach, Germany) was solubilized in bidistilled deionized water $(5 \mathrm{~mL} ; 5 \mathrm{mg} / \mathrm{mL})$ and immediately added to the HFn solution at a final dye concentration of 1 $\mathrm{mg} / \mathrm{mL}$. The mixture was incubated for $2 \mathrm{~h}$ at RT to allow complete refolding of the HFn quaternary structure. The resulting HFn-ICG nanoparticles were then concentrated by means of Amicon Ultra-4 centrifugal filter devices (Merck S.p.a., Milan, Italy, Catalog Number: UFC810024). Unloaded ICG has been removed by gel filtration using a Zeba Spin Desalting column (Thermo Fisher Scientific, Monza, Italy; Catalog Number: 89890).

Transmission Electron Microscopy. A drop of HFn suspension was dried on the Formvar net at RT, stained with uranil-acetate $1 \%$ for $30 \mathrm{~s}$ at RT and dried over night at RT. Samples were evaluated by TEM (Tecnai Spirit, FEI). Magnification 300,000 $\times$ and 80,000 $\times$. High-magnifications images were further elaborated with the Profile Plot ImageJ software tool, to measure the size of HFn-ICG nanocages.
Raman Spectroscopy. Raman spectra were recorded using an inVia Raman microscope from Renishaw (UK) equipped with laser light sources operating at $785 \mathrm{~nm}$. The Raman spectrometer was calibrated daily using the band of monocrystalline silicon at $520.7 \mathrm{~cm}^{-1}$. Raman spectra were acquired from $12 \mu \mathrm{L}$ drops of water solution of free ICG and of HFn-ICG dried on top of a $\mathrm{CaF}_{2}$ slide (Crystran, UK) without any further preparation. Spectra were collected using a $785 \mathrm{~nm}$ laser line of $6.25 \mathrm{~mW}$ focused on the sample using a $100 \times$ objective for $10 \mathrm{~s}$. Shown spectra are the averaging of six acquisitions after baseline subtraction and vector normalization.

Analysis of ICG Fluorescence Properties and Quantification of ICG Encapsulation Efficiency. The fluorescence properties of free and encapsulated ICG were studied in different media by fluorescence spectroscopy (FP8300 spectrofluorometer equipped with a FMP-825 multiwell reader; Jasco, Cremella, LC, Italy). Fluorescence yield and emission spectrum of the dye were studied in $\mathrm{ddH}_{2} \mathrm{O}, \mathrm{PBS}$, DMEM, human plasma, and milk. This analysis was mandatory to select a solvent suitable for further quantitative analysis of ICG encapsulation in HFN nanocages. We selected milk for its high fluorescence yield and data reproducibility.

A standard curve was obtained by fluorescence analysis of different concentrations of free ICG in milk at different concentrations $(1.7,10,20,40,80$, and $160 \mu \mathrm{M})$ and was used to quantify the amount of ICG loaded inside the HFn nanocages. To avoid issues due to differences in fluorescence properties between free and encapsulated ICG, the dye was first extracted from the nanocages and then quantified. To this aim, $80 \mu \mathrm{L}$ of cold acetonitrile was added to $20 \mu \mathrm{L}$ of the HFn-ICG sample. The mixture was vortexed and centrifuged at $14,000 \mathrm{rpm}$ for $10 \mathrm{~min}$ in order to precipitate denatured HFn and release encapsulated ICG in solution. Then, different dilutions were prepared to avoid quenching effect, and fluorescence emission of the extract was measured with an excitation wavelength of $730 \mathrm{~nm}$. Results were interpolated with the standard curve to calculate the amount of encapsulated ICG. Experiments were performed in triplicate.

Kinetics of Spontaneous ICG Release In Vitro. HFnICG was loaded in a dialysis device (Float-A-lyzer G2 Dialysis Device MWCO: $100 \mathrm{KD}$, Spectrum Labs, Compton, CA, USA) and kept in a water bath at $4{ }^{\circ} \mathrm{C}$ for 14 days. At predetermined time points (15 min, 1, 2, 3, 18, 24, 48, 72, 96 h, $6,7,8$, and 14 days), $5 \mathrm{~mL}$ of buffer was collected, replaced with fresh buffer in order to maintain the sink condition, and the fluorescence of each collected sample was measured by spectrofluorimetry. Moreover, the decay in fluorescence of HFn-ICG was studied in parallel for the whole duration of the dialysis. To calculate the relative amount of released ICG at each time point, the fluorescence value was interpolated with the calibration curve, as already described, and normalized with the fluorescence decay measured throughout the dialysis period (data not shown). This allowed us to obtain 100\% recovery of the initially loaded ICG. Fluorescence was measured $\left(\lambda_{\text {exc }}:=760 \mathrm{~nm}, \lambda_{\text {emis }}:=780 \mathrm{~nm}\right)$. All studies were performed in triplicate.

Cell Culture. HT-29, HCT116, SNU-16, MCF7, MDA-MB 468, MDA-MB 231, HCC1937, and MCF-10A cell lines were purchased by ATCC-LGC Standards. HT-29, HCT116, SNU16, and HCC1937 cells were cultured in RPMI 1640 Medium. MDA-MB 468 and MCF7 cells in high glucose DMEM. MDAMB 231 cells were cultured in minimum essential medium. All 
media were supplemented with $10 \%$ heat inactivated fetal bovine serum (FBS), $2 \mathrm{mM}$ L-glutamine, $100 \mathrm{U} \mathrm{mL}^{-1}$ penicillin, and $0.1 \mathrm{mg} / \mathrm{mL}$ streptomycin, except for HT-29 and HCT116 cells that were cultured in medium supplemented with $10 \%$ heat inactivated FBS, $1.5 \mathrm{mM}$ L-glutamine, $100 \mathrm{U} \mathrm{mL}^{-1}$ penicillin, and $0.1 \mathrm{mg} / \mathrm{mL}$ streptomycin. MCF$10 \mathrm{~A}$ was cultured in MEBM medium supplemented with MEGM kit from Lonza (CC-3150). All cell lines grew at $37^{\circ} \mathrm{C}$ in a humidified atmosphere containing $5 \% \mathrm{CO}_{2}$ and were subcultured prior to confluence using trypsin/EDTA. Cell culture medium and chemicals for cell culture were purchased from Euroclone.

TfR1 Expression. HT-29, HCT116, SNU-16, MCF7, MDA-MB 468, MDA-MB 231, and HCC1937 cells $(5 \times$ $\left.10^{5}\right)$ were labeled with anti-TfR1 antibody diluted 1:100 [1 $\mu \mathrm{g} /$ tube; CD71 antibody (clone ICO-92), Thermo Fisher Scientific, Catalog Number \#: MA1-7657] in blocking buffer (PBS, 2\%) bovine serum albumin [BSA; Sigma-Aldrich S.r.l., Milan, Italy, and $2 \%$ goat serum (Euroclone S.p.A., Pero, Italy)] for $15 \mathrm{~min}$ at RT and cells were washed three times with PBS. Then, cells were labeled with Alexa Fluor 488 goat anti-mouse secondary antibody diluted 1:200 (1 $\mu \mathrm{L} /$ tube; Thermo Fisher Scientific, Monza, Italy; Catalog Number \#: A11001 ) in blocking buffer for $15 \mathrm{~min}$ at RT and were washed thrice with PBS before analysis using CytoFLEX flow cytometer (Beckman Coulter, Cassina De' Pecchi, Italy), and 20,000 events were acquired, after gating on viable cells and on singlets. Cells immunodecorated only with the secondary antibody were used to set the region of positivity.

In Tube-Cell Binding Assay at $4{ }^{\circ} \mathrm{C}$. Cells $\left(5 \times 10^{5}\right)$ were collected and incubated for $2 \mathrm{~h}$ at $4{ }^{\circ} \mathrm{C}$ in blocking buffer (PBS, 0.3\% BSA) supplemented with 20,50 , and $100 \mu \mathrm{g} / \mathrm{mL}$ of FITC-labeled HFn. After incubation, cells were washed three times with PBS, suspended in $0.5 \mathrm{~mL}$ of PBS, and analyzed using a CytoFLEX flow cytometer (Beckman Coulter, Cassina De' Pecchi, Italy). 20,000 events were acquired for each analysis, after gating on viable cells and on singlets. A sample of untreated cells was used to set the appropriate gates.

Competition Assay. HT-29 cells $\left(5 \times 10^{5}\right)$ were collected and preincubated for $2 \mathrm{~h}$ at $4{ }^{\circ} \mathrm{C}$ in $500 \mu \mathrm{L}$ of $0.3 \%$ BSA-PBS with or without $1 \mathrm{mg} / \mathrm{mL}$ of free unlabeled $\mathrm{HFn}$ as competitor. Then, $20 \mu \mathrm{g} / \mathrm{mL}$ of FITC-labeled HFn was added for $1 \mathrm{~h}$ at 4 ${ }^{\circ} \mathrm{C}$. Cells were washed three times with PBS and analyzed by CytoFLEX. After gating on viable and single cells, 20,000 events were acquired for each analysis. Untreated cells were used to set the positivity region.

Confocal Laser Scanning Microscopy. Cells $\left(2 \times 10^{5}\right)$ were cultured until subconfluence on cover glass slides precoated with collagen and incubated with HFn-ICG at a concentration of for $2 \mathrm{~h}$ at $4{ }^{\circ} \mathrm{C}$. After incubation, cells were washed three times with PBS, fixed for 5 min with $4 \%$ paraformaldehyde (Sigma-Aldrich), and washed thrice with PBS. A blocking step was executed for $1 \mathrm{~h}$ at RT with a solution containing 2\% BSA (Sigma-Aldrich) and 2\% goat serum (Euroclone) in PBS. Membranes were labeled incubating coverslips with Alexa Fluor 488 wheat germ agglutinin (Life Technologies) diluted 1:200 in PBS for 10 min. TfR1 colocalization was revealed with the anti-TfR1 antibody (1:200; ab84036; Abcam) and recognized by Alexa Fluor 488-conjugated antibody against rabbit IgGs (Thermo Fischer Scientific) at a 1:300 dilution by incubating for $2 \mathrm{~h}$ at RT in PBS, $2 \%$ BSA, $2 \%$ goat serum, and $0.2 \mu \mathrm{g} / \mathrm{mL}$ DAPI (4',6-diamino-2-phenylindole; Thermo Fischer Scientific).
Coverslips were mounted in Prolong Gold antifade reagent (Thermo Fisher Scientific). Microscopy analysis was performed with the Leica SP8 system equipped with laser excitation lines at 405, 488, 552, and $633 \mathrm{~nm}$, using a $63 \times$ magnification oil immersion lens.

To evaluate kinetics of internalization of HFn-ICG and free ICG, MDA-MB 231 cells were cultured until subconfluence on cover glass slides precoated with collagen and incubated for 15 min, $1,3,24$, and $48 \mathrm{~h}$ at $37^{\circ} \mathrm{C}$ in complete cell culture medium supplemented with HFn-ICG nanoparticles or ICG free (green; $50 \mu \mathrm{g} / \mathrm{mL}$ ). After incubation at the indicated time points, cells were washed with PBS, fixed for $5 \mathrm{~min}$ with $4 \%$ paraformaldehyde (Sigma-Aldrich), and then treated for $5 \mathrm{~min}$ with $0.1 \%$ Triton X-100 (Sigma-Aldrich). Subsequently, a blocking step was performed for $1 \mathrm{~h}$ at RT with a solution containing 2\% BSA (Sigma-Aldrich), 2\% goat serum (Euroclone), and $0.2 \mu \mathrm{g} / \mathrm{mL}$ DAPI (Thermo Fischer Scientific) in PBS. TfR1 staining was performed with the anti-TfR1 antibody (1:200; ab84036; Abcam) recognized by Alexa Fluor 488conjugated antibody against rabbit IgGs (Thermo Fischer Scientific) at a 1:300 dilution by incubating for $2 \mathrm{~h}$ at RT in PBS, $2 \%$ BSA, and $2 \%$ goat serum. Coverslips were mounted in Prolong Gold antifade reagent (Thermo Fisher Scientific), and images were acquired with a Leica SP8 microscope confocal system equipped with laser excitation lines 405, 488, 535, and $633 \mathrm{~nm}$. Images were acquired with $63 \times$ magnification oil immersion lens.

Cellular Uptake. Cells $\left(1 \times 10^{3}\right)$ were seeded in a black 96-well plate in order to inhibit influence of background fluorescence during analysis. The day after seeding, cells were treated with $50 \mu \mathrm{g} / \mathrm{mL}$ of HFn-ICG for different time periods (15 min, 1, 2, 3, 4, 6, 24, 48, and $72 \mathrm{~h}$ ) at $37^{\circ} \mathrm{C}$. After incubation, cells were washed five times with PBS and then $100 \mu \mathrm{L}$ of cold acetonitrile were added to each well for $24 \mathrm{~h}$ at $-20{ }^{\circ} \mathrm{C}$ in order to lyse cells and extract ICG. After cell lysis, a dilution 1:2 in milk of each well suspension was made. Plate fluorescence has been measured using the IVIS Lumina II imaging system (PerkinElmer, Waltham, MA, USA). The following acquisition parameters were used: excitation filter $745 \mathrm{~nm}$, emission filter ICG, exposure time $=30 \mathrm{~s}$, field of view $=12.5 \times 12.5 \mathrm{~cm}$, binning factor $=2$, and $f /$ stop $=2$. Image processing and analysis were done using Living Image 4.3.1 software (PerkinElmer). The fluorescence specific signal was shown as the radiant efficiency (emission light [photons/sec/ $\left.\mathrm{cm}^{2} / \mathrm{str}\right]$ /excitation light $\left.\left[\mu \mathrm{W} / \mathrm{cm}^{2}\right]\right)$. The fluorescence signal for each individual well was counted by selecting region of interest (ROI) and quantifying as the total radiant efficiency ([photons $\left./ \mathrm{sec}] /\left[\mu \mathrm{W} / \mathrm{cm}^{2}\right]\right)$.

In Vivo Labeling of Primary Tumor. Seven weeks old female BALB/c mice were injected into the mammary fat pad with 100,000 4T1-Luc cells (Bioware Ultra, PerkinElmer). After 10 days, mice were intravenously injected with ICG or HFn-ICG (3.8 mg/Kg). ICG distribution and tumor accumulation were monitored for $24 \mathrm{~h}$ using the in vivo imaging IVIS Lumina II (PerkinElmer) instrument and the KARL STORZ NIR/ICG endoscopic system (OPAL1 Technology, equipped with an high-end full $\mathrm{HD}$ camera IMAGE 1 SPIES and a xenon light source D-light P SCB; KARL STORZ GmbH \& Co. KG, Tuttlingen, Germany). Reported images were acquired 6 and $24 \mathrm{~h}$ after injection, when differences between free and encapsulated ICG were most evident. Animals have been managed according to 
procedures approved by Italian Ministry of Health (aut. Number 611/2019-PR).

\section{ASSOCIATED CONTENT}

\section{(s) Supporting Information}

The Supporting Information is available free of charge at https://pubs.acs.org/doi/10.1021/acsomega.0c00244.

HFn-F binding in MCF-10A cells, fluorescence emission of ICG, standard fluorescence curve of free ICG, ICG release from HFn-ICG, colocalization of HFn-ICG and TfR1, and in vivo tumor targeting of HFn-ICG and ICG (PDF)

\section{AUTHOR INFORMATION}

\section{Corresponding Authors}

Fabio Corsi - Nanomedicine Laboratory, Department of Biomedical and Clinical Sciences "Luigi Sacco", Università degli Studi di Milano, 20157 Milan, Italy; Breast Unit and Nanomedicine and Molecular Imaging Lab, Istituti Clinici Scientifici Maugeri IRCCS, 27100 Pavia (PV), Italy; Email: fabio.corsi@unimi.it

Serena Mazzucchelli - Nanomedicine Laboratory, Department of Biomedical and Clinical Sciences "Luigi Sacco", Università degli Studi di Milano, 20157 Milan, Italy; (1) orcid.org/00000001-6904-8895; Email: serena.mazzucchelli@unimi.it

\section{Authors}

Leopoldo Sitia - Nanomedicine Laboratory, Department of Biomedical and Clinical Sciences "Luigi Sacco", Università degli Studi di Milano, 20157 Milan, Italy

Marta Sevieri - Nanomedicine Laboratory, Department of Biomedical and Clinical Sciences "Luigi Sacco", Università degli Studi di Milano, 20157 Milan, Italy

Arianna Bonizzi - Nanomedicine Laboratory, Department of Biomedical and Clinical Sciences "Luigi Sacco", Università degli Studi di Milano, 20157 Milan, Italy

Raffaele Allevi - Nanomedicine Laboratory, Department of Biomedical and Clinical Sciences "Luigi Sacco", Università degli Studi di Milano, 20157 Milan, Italy

Carlo Morasso - Nanomedicine and Molecular Imaging Lab, Istituti Clinici Scientifici Maugeri IRCCS, 27100 Pavia (PV), Italy

Diego Foschi - General Surgery Division, Department of Biomedical and Clinical Sciences "Luigi Sacco", Università degli Studi di Milano, 20157 Milan, Italy

Complete contact information is available at:

https://pubs.acs.org/10.1021/acsomega.0c00244

\section{Author Contributions}

S.M., D.F. and F.C. conceived, designed, and supervised the study. A.B. and M.S. developed HFn-ICG. M.S., A.B., and L.S. performed in vitro studies to evaluate interaction and uptake in CCs. R.A. performed transmission electron microscopy, while C.M. recorded Raman spectra. L.S. quantified encapsulated ICG. S.M. and L.S. analyzed the data. S.M., L.S., M.S., and F.C. wrote the manuscript. S.M., L.S., and F.C. revised the paper. Authorship must be limited to those who have contributed substantially to the work reported. All authors have given approval to the final version of the manuscript.

\section{Funding}

This work was supported by University of Milan, Department of Biomedical and Clinical Sciences "L. Sacco", 14831RV_PRO_RIC16DFOSC_M.

\section{Notes}

The authors declare no competing financial interest.

\section{ACKNOWLEDGMENTS}

We acknowledge the Pediatric Clinical Research Center "Romeo and Enrica Invernizzi" at University of Milan for imaging facility and for SM position, and KARL STORZ $\mathrm{GmbH} \&$ Co for NIR/ICG endoscopic system.

\section{ABBREVIATIONS}

ICG, indocyanine green; NIR, near infrared; FGS, imageguided surgery; LNM, lymph node mapping; EPR, enhanced permeation effect; $\mathrm{HFn}, \mathrm{H}$-ferritin; TfR1, transferrin receptor1; HFn-ICG, ICG loaded HFn nanocages; CC, cancer cell; BSA, bovine serum albumin; HFn-F, FITC conjugated HFn nanocages; RT, room temperature; TEM, transmission electron microscopy; DMEM, Dulbecco's modified Eagle media; ultrapure bidistilled-Milli-Q water, $\mathrm{ddH}_{2} \mathrm{O}$; PBS, phosphate buffered solution; ROI, region of interest

\section{REFERENCES}

(1) Hill, T. K.; Abdulahad, A.; Kelkar, S. S.; Marini, F. C.; Long, T. E.; Provenzale, J. M.; Mohs, A. M. Indocyanine Green-Loaded Nanoparticles for Image-Guided Tumor Surgery. Bioconjugate Chem. 2015, 26, 294-303.

(2) Maarek, J.-M. I.; Holschneider, D. P.; Harimoto, J.; Yang, J.; Scremin, O. U.; Rubinstein, E. H. Measurement of Cardiac Output with Indocyanine Green Transcutaneous Fluorescence Dilution Technique. Anesthesiology 2004, 100, 1476-1483.

(3) Alander, J. T.; Kaartinen, I.; Laakso, A.; Pätilä, T.; Spillmann, T.; Tuchin, V. V.; Venermo, M.; Välisuo, P. A Review of Indocyanine Green Fluorescent Imaging in Surgery. Int. J. Biomed. Imaging 2012, $2012,1-26$.

(4) Sakka, S. G. Assessing Liver Function. Curr. Opin. Crit. Care 2007, 13, 207-214.

(5) Wang, H.; Li, X.; Tse, B. W.-C.; Yang, H.; Thorling, C. A.; Liu, Y.; Touraud, M.; Chouane, J. B.; Liu, X.; Roberts, M. S.; et al. Indocyanine Green-Incorporating Nanoparticles for Cancer Theranostics. Theranostics 2018, 8, 1227-1242.

(6) Giuliano, A. E.; Kirgan, D. M.; Guenther, J. M.; Morton, D. L. Lymphatic Mapping and Sentinel Lymphadenectomy for Breast Cancer. Ann. Surg. 1994, 220, 391-401. ; discussion 398-401

(7) Verbeek, F. P. R.; Troyan, S. L.; Mieog, J. S. D.; Liefers, G.-J.; Moffitt, L. A.; Rosenberg, M.; Hirshfield-Bartek, J.; Gioux, S.; van de Velde, C. J. H.; Vahrmeijer, A. L.; et al. Near-Infrared Fluorescence Sentinel Lymph Node Mapping in Breast Cancer: A Multicenter Experience. Breast Cancer Res. Treat. 2014, 143, 333-342.

(8) Veronesi, U.; Paganelli, G.; Galimberti, V.; Viale, G.; Zurrida, S.; Bedoni, M.; Costa, A.; de Cicco, C.; Geraghty, J. G.; Luini, A.; et al. Sentinel-Node Biopsy to Avoid Axillary Dissection in Breast Cancer with Clinically Negative Lymph-Nodes. Lancet 1997, 349, 18641867.

(9) Clinicaltrials.Gov. Indocyanine Green. https://clinicaltrials.gov/ ct $2 /$ results? cond $=$ Cancer\&term $=$ indocyanine + green $+\&$ type $=\&$ rslt $=$ \&age_v=\&gndr=\&intr=\&titles=\&outc=\&spons=\&lead=\&id=\&cntry= \&state $=\&$ city $=\&$ dist $=\& l o c n=\& \operatorname{rsub}=\& \operatorname{strd} s=\& \operatorname{strd} e=\& \operatorname{prcd} s=$ \&prcd_e $=\& s f p d \_s=\& s f p d \_e=\& \operatorname{rfpd} s=\& r f p d \_e=\& l u p d \_s=\& l u p d$ $\mathrm{e}=\&$ sort $=$.

(10) Mondal, S. B.; Gao, S.; Zhu, N.; Liang, R.; Gruev, V.; Achilefu, S. Chapter Five - Real-Time Fluorescence Image-Guided Oncologic Surgery. In Advances in Cancer Research; Pomper, M. G., Fisher, P. B., 
Eds.; Emerging Applications of Molecular Imaging to Oncology; Academic Press, 2014; Vol. 124, pp 171-211.

(11) Veys, I.; Pop, F.-C.; Vankerckhove, S.; Barbieux, R.; Chintinne, M.; Moreau, M.; Nogaret, J.-M.; Larsimont, D.; Donckier, V.; Bourgeois, P.; et al. ICG-Fluorescence Imaging for Detection of Peritoneal Metastases and Residual Tumoral Scars in Locally Advanced Ovarian Cancer: A Pilot Study. J. Surg. Oncol. 2018, 117, 228-235.

(12) Veys, I.; Pop, C.-F.; Barbieux, R.; Moreau, M.; Noterman, D.; De Neubourg, F.; Nogaret, J.-M.; Liberale, G.; Larsimont, D.; Bourgeois, P. ICG Fluorescence Imaging as a New Tool for Optimization of Pathological Evaluation in Breast Cancer Tumors after Neoadjuvant Chemotherapy. PLoS One 2018, 13, No. e0197857.

(13) Kirchherr, A.-K.; Briel, A.; Mäder, K. Stabilization of Indocyanine Green by Encapsulation within Micellar Systems. Mol. Pharm. 2009, 6, 480-491.

(14) Lee, Y.-H.; Chang, D.-S. Fabrication, Characterization, and Biological Evaluation of Anti-HER2 Indocyanine Green-DoxorubicinEncapsulated PEG-b-PLGA Copolymeric Nanoparticles for Targeted Photochemotherapy of Breast Cancer Cells. Sci. Rep. 2017, 7, 46688.

(15) Liu, F.; Chen, Y.; Li, Y.; Guo, Y.; Cao, Y.; Li, P.; Wang, Z.; Gong, Y.; Ran, H. Folate-Receptor-Targeted Laser-Activable Poly(Lactide-Co-Glycolic Acid) Nanoparticles Loaded with Paclitaxel/ Indocyanine Green for Photoacoustic/Ultrasound Imaging and Chemo/Photothermal Therapy. Int. J. Nanomed. 2018, 13, 51395158.

(16) Chen, H.-H.; Lu, I.-L.; Liu, T.-I.; Tsai, Y.-C.; Chiang, W.-H.; Lin, S.-C.; Chiu, H.-C. Indocyanine Green/Doxorubicin-Encapsulated Functionalized Nanoparticles for Effective Combination Therapy against Human MDR Breast Cancer. Colloids Surf., B 2019, 177, 294305 .

(17) Zheng, X.; Xing, D.; Zhou, F.; Wu, B.; Chen, W. R. Indocyanine Green-Containing Nanostructure as Near Infrared Dual-Functional Targeting Probes for Optical Imaging and Photothermal Therapy. Mol. Pharm. 2011, 8, 447-456.

(18) Miranda, D.; Wan, C.; Kilian, H. I.; Mabrouk, M. T.; Zhou, Y.; Jin, H.; Lovell, J. F. Indocyanine Green Binds to DOTAP Liposomes for Enhanced Optical Properties and Tumor Photoablation. Biomater. Sci. 2019, 7, 3158-3164.

(19) Ichihara, H.; Okumura, M.; Tsujimura, K.; Matsumoto, Y. Theranostics with Hybrid Liposomes in an Orthotopic Graft Model Mice of Breast Cancer. Anticancer Res. 2018, 38, 5645-5654.

(20) Akhtar, M. J.; Ahamed, M.; Alhadlaq, H. A.; Alrokayan, S. A.; Kumar, S. Targeted Anticancer Therapy: Overexpressed Receptors and Nanotechnology. Clin. Chim. Acta 2014, 436, 78-92.

(21) Holt, D.; Okusanya, O.; Judy, R.; Venegas, O.; Jiang, J.; DeJesus, E.; Eruslanov, E.; Quatromoni, J.; Bhojnagarwala, P.; Deshpande, C.; et al. Intraoperative Near-Infrared Imaging Can Distinguish Cancer from Normal Tissue but Not Inflammation. PLoS One 2014, 9, No. e103342.

(22) Holzer, W.; Mauerer, M.; Penzkofer, A.; Szeimies, R.-M.; Abels, C.; Landthaler, M.; Bäumler, W. Photostability and Thermal Stability of Indocyanine Green. J. Photochem. Photobiol., B 1998, 47, 155-164. (23) Mordon, S.; Devoisselle, J. M.; Soulie-Begu, S.; Desmettre, T. Indocyanine Green: Physicochemical Factors Affecting Its Fluorescencein vivo. Microvasc. Res. 1998, 55, 146-152.

(24) Khoshnejad, M.; Parhiz, H.; Shuvaev, V. V.; Dmochowski, I. J.; Muzykantov, V. R. Ferritin-Based Drug Delivery Systems: Hybrid Nanocarriers for Vascular Immunotargeting. J. Controlled Release 2018, 282, 13-24.

(25) Zang, J.; Zheng, B.; Zhang, X.; Arosio, P.; Zhao, G. Design and Site-Directed Compartmentalization of Gold Nanoclusters within the Intrasubunit Interfaces of Ferritin Nanocage. J. Nanobiotechnol. 2019, 17,79 .

(26) Truffi, M.; Fiandra, L.; Sorrentino, L.; Monieri, M.; Corsi, F.; Mazzucchelli, S. Ferritin Nanocages: A Biological Platform for Drug Delivery, Imaging and Theranostics in Cancer. Pharmacol. Res. 2016, 107, 57-65.
(27) Zhen, Z.; Tang, W.; Guo, C.; Chen, H.; Lin, X.; Liu, G.; Fei, B.; Chen, X.; Xu, B.; Xie, J. Ferritin Nanocages To Encapsulate and Deliver Photosensitizers for Efficient Photodynamic Therapy against Cancer. ACS Nano 2013, 7, 6988-6996.

(28) Fan, K.; Cao, C.; Pan, Y.; Lu, D.; Yang, D.; Feng, J.; Song, L.; Liang, M.; Yan, X. Magnetoferritin Nanoparticles for Targeting and Visualizing Tumour Tissues. Nat. Nanotechnol. 2012, 7, 459-464.

(29) Wang, Z.; Gao, H.; Zhang, Y.; Liu, G.; Niu, G.; Chen, X. Functional Ferritin Nanoparticles for Biomedical Applications. Front. Chem. Sci. Eng. 2017, 11, 633-646.

(30) Mazzucchelli, S.; Bellini, M.; Fiandra, L.; Truffi, M.; Rizzuto, M. A.; Sorrentino, L.; Longhi, E.; Nebuloni, M.; Prosperi, D.; Corsi, F. Nanometronomic Treatment of 4T1 Breast Cancer with Nanocaged Doxorubicin Prevents Drug Resistance and Circumvents Cardiotoxicity. Oncotarget 2017, 8, 8383.

(31) Mazzucchelli, S.; Truffi, M.; Baccarini, F.; Beretta, M.; Sorrentino, L.; Bellini, M.; Rizzuto, M. A.; Ottria, R.; Ravelli, A.; Ciuffreda, P.; et al. H-Ferritin-Nanocaged Olaparib: A Promising Choice for Both BRCA-Mutated and Sporadic Triple Negative Breast Cancer. Sci. Rep. 2017, 7, 7505.

(32) Bonizzi, A.; Truffi, M.; Sevieri, M.; Allevi, R.; Sitia, L.; Ottria, R.; Sorrentino, L.; Sottani, C.; Negri, S.; Grignani, E.; et al. Everolimus Nanoformulation in Biological Nanoparticles Increases Drug Responsiveness in Resistant and Low-Responsive Breast Cancer Cell Lines. Pharmaceutics 2019, 11, 384.

(33) Pandolfi, L.; Bellini, M.; Vanna, R.; Morasso, C.; Zago, A.; Carcano, S.; Avvakumova, S.; Bertolini, J. A.; Rizzuto, M. A.; Colombo, M.; et al. H-Ferritin Enriches the Curcumin Uptake and Improves the Therapeutic Efficacy in Triple Negative Breast Cancer Cells. Biomacromolecules 2017, 18, 3318-3330.

(34) Huang, P.; Rong, P.; Jin, A.; Yan, X.; Zhang, M. G.; Lin, J.; Hu, H.; Wang, Z.; Yue, X.; Li, W.; et al. Dye-Loaded Ferritin Nanocages for Multimodal Imaging and Photothermal Therapy. Adv. Mater. 2014, 26, 6401-6408.

(35) Bellini, M.; Mazzucchelli, S.; Galbiati, E.; Sommaruga, S.; Fiandra, L.; Truffi, M.; Rizzuto, M. A.; Colombo, M.; Tortora, P.; Corsi, F.; et al. Protein Nanocages for Self-Triggered Nuclear Delivery of DNA-Targeted Chemotherapeutics in Cancer Cells. J. Controlled Release 2014, 196, 184-196.

(36) Corsi, F.; Mazzucchelli, S. The Potential of Protein-Based Nanocages for Imaging and Drug Delivery. Ther. Delivery 2016, 7, 149-151.

(37) Hollins, B.; Noe, B.; Henderson, J. M. Fluorometric Determination of Indocyanine Green in Plasma. Clin. Chem. 1987, 33, 765-768.

(38) Li, L.; Fang, C. J.; Ryan, J. C.; Niemi, E. C.; Lebrón, J. A.; Björkman, P. J.; Arase, H.; Torti, F. M.; Torti, S. V.; Nakamura, M. C.; et al. Binding and Uptake of H-Ferritin Are Mediated by Human Transferrin Receptor-1. Proc. Natl. Acad. Sci. U.S.A. 2010, 107, 35053510.

(39) Watts, C. Rapid Endocytosis of the Transferrin Receptor in the Absence of Bound Transferrin. J. Cell Biol. 1985, 100, 633-637.

(40) Mayle, K. M.; Le, A. M.; Kamei, D. T. The Intracellular Trafficking Pathway of Transferrin. Biochim. Biophys. Acta, Gen. Subj. 2012, 1820, 264-281.

(41) Tan, T.; Wang, H.; Cao, H.; Zeng, L.; Wang, Y.; Wang, Z.; Wang, J.; Li, J.; Wang, S.; Zhang, Z.; Li, Y. Deep Tumor-Penetrated Nanocages Improve Accessibility to Cancer Stem Cells for Photothermal-Chemotherapy of Breast Cancer Metastasis. Adv. Sci. 2018, 5, 1801012.

(42) Alander, J. T.; Kaartinen, I.; Laakso, A.; Pätilä, T.; Spillmann, T.; Tuchin, V. V.; Venermo, M.; Välisuo, P. A review of indocyanine green fluorescent imaging in surgery. Int. J. Biomed. Imaging 2012, 2012, 940585. 